\title{
KINESITHERAPEUTIC INFLUENCE ON PHYSICAL TOLERANCE AFTER TRANSCATHETER AORTIC VALVE IMPLANTATION
}

\author{
Zhasmina Koleva \& Antoaneta Dimitrova \\ National Sports Academy "Vassil Levski", Sofia, Bulgaria
}

\begin{abstract}
Cardiac rehabilitation is a customized in-hospital and outpatient program of therapeutic exercises and education, designed to improve the recovery of patients with a various heart diseases, cardiac surgery or implantation.

The aim of the present study is to evaluate the effect of three months of kinesitherapy on the functional mobility in patients post transcatheter aortic valve implantation (TAVI). 31 patients were referred for in-hospital and home based kinesitherapy for three months after TAVI. The subjects underwent four assessments in-hospital and after discharge to evaluate their functional abilities, muscle strength and muscle endurance with the following tests six minutes walking test (6MWT), five times sit-to-stand test (FTSTS), Borg scale and dynamometry of the upper limbs.

All patients had significant improvement in their physical tolerance. The time for performing the FTSTS test decreased with $3 \mathrm{sec}$ on the $3^{\text {rd }}$ month, which indicates improvement in their functional lower limb muscle strength. Positive changes in muscle strength of the upper limbs were also noted.

The kinesitherapeutic program benefits all the patients after TAVI in regards to their walking performance and their upper and lower limbs muscle strength.
\end{abstract}

Key words: exercise therapy, cardiology, geriatric

\section{INTRODUCTION}

Transcatheter aortic valve implantation (TAVI) is an alternative intervention mainly for elderly patients with contraindications to surgical aortic valve replacement. Cardiac rehabilitation (CR) is a well-established therapeutic approach for people with cardiac disease. Regular physical activity is an essential part of treatment in geriatric patients. It demonstrates preventative and therapeutic effects on many chronic conditions (Fauchere, et al., 2014).

Patients after TAVI, are candidates for referral to exercise-based patient-centered kinesitherapy (KT) to reduce prolonged limitation in functional abilities and to increase independency for daily life activities and participation in socio-cultural life (Ribeiro, et al., 2017). To ensure better treatment plan and positive results, many components should be taken into consideration in the assessment of patients, as comorbidities, physical condition, motivation etc.

The aim of the present study is to evaluate the effect of three months of KT on the functional mobility in patients after TAVI.

\section{METHODS}

31 patients (15 male, 16 female; mean age $78,4 \pm 3,1$; NYHA III-IV before TAVI) referred for KT underwent 4 assessments in-hospital and after discharge to evaluate the muscle strength and muscle endurance with the following tests - five times sitto-stand test (FTSTS), Borg scale and dynamometry of the upper limbs. The assessments were performed before TAVI procedure, on the day of hospital discharge, on the 1st and 3rd month after TAVI procedure.

The Borg Scale assesses the intensity of breathlessness during the physical exertion. It is a 10 -point scale ( $0-10$ points) for subjectively determining the level of exertion. The higher the number of points, the greater is the feeling of shortness of breath (Borg, 1982). The timing of FTSTS test performance is important for the patient's ability to perform functional activities and for the functional strength of their lower limbs. Normative values are $11,4 \sec (60-69$ years), 12,6 sec (70-79 years) and $14,8 \mathrm{sec}$ ( $80-89$ years) (Bohannon, 2006). The grip strength was measured with a conventional electronic hand dynamometer EH101, with the patient in a standing position with their arm extended straight down on the side of the body. 
Three attempts are made for each hand and the best result is taken into consideration in the results. In our study the right hand was the dominant for all subjects. The patients enrolled in the current study, were chosen based on the following inclusion criteria (TAVI patients above the age of 70; male or female; signed informed consent) and exclusion criteria (unstable clinical condition - according to treating physician; handicap before the procedure; recent stroke; decompensate diabetes mellitus type II; severe cognitive decline). Patients' sociodemographic and concomitant diseases' characteristics were collected at the beginning of the study [Table 1].

Table 1. Baseline characteristics of the patients before implantation

\begin{tabular}{|l|l|}
\hline \multicolumn{1}{|c|}{ Parameters } & TAVI n=31 \\
\hline Age $($ years $)$ mean \pm SD & $78,4 \pm 3,1$ \\
\hline Male $-\mathrm{n}$ & 15 \\
\hline Female $-\mathrm{n}$ & 16 \\
BMI male $\left(\mathrm{kg} / \mathrm{m}^{2}\right)$ & $23,9 \pm 0,8$ \\
BMI female $\left(\mathrm{kg} / \mathrm{m}^{2}\right)$ & $24,8 \pm 1,2$ \\
\hline NYHA class & $3,2 \pm 0,2$ \\
\hline Cumulative Illness Rating Scale & $10,9 \pm 3,9$ \\
\hline COMORBIDITIES N & \\
\hline CHD & 31 \\
\hline Peripheral vascular disease & 8 \\
\hline Arterial hypertension & 28 \\
\hline Pulmonary diseases & 7 \\
\hline Renal diseases & 12 \\
\hline Diabetes mellitus & 8 \\
\hline Gastrointestinal diseases & 14 \\
\hline Anaemia & 10 \\
\hline Osteoarthrosis & 10 \\
\hline PREVIOUS DISEASES N & \\
\hline Prev MI & 2 \\
\hline Prev PCI & 2 \\
\hline Prev CABG & 7 \\
\hline Stroke/TIA & 4 \\
\hline SMOKING STATUS N & \\
\hline Current smoker & 7 \\
\hline Smoking history & 17 \\
\hline Never smoked & 7 \\
\hline
\end{tabular}

SD-standard deviation; BMI-Body Mass Index; NYHA-New York Heart Association; CHD-chronic ishaemic heart disease; MI-myocardial infarction; PCI-percutaneous coronary intervention; CABG-coronary artery bypass graft; TIA- Transient Ischemic Attack

Exercise protocol for in-hospital kinesitherapy

Exercise protocol for in-hospital KT was performed twice daily, with low intensity (40-50\% HR max), duration from $10-15 \mathrm{~min}$ to $30-45 \mathrm{~min}$, on average for 10 days. KT was individually tailored, in connection with the patient's goals for everyday life activities. The protocol included educating the patients on proper mobility and performance of daily life activities, mobilization to sitting, standing and slow walking at least six minutes daily; rhythmic exercises for the distal parts of the limbs, exercises for gradually strengthening the upper and the lower limbs, diaphragmatic and chest breathing, breathing with a device (Coach2 Incentive Spirometer) for inspiratory training, isometric exercises in circular mode. The feedback breathing device for inspiratory training was applied to all patients with the instruction to make 5 repetitions with 1 to $3 \mathrm{~min}$ rests between them, at least 4-5 times a day, every day of the week during hospital stay (Grigorova-Petrova, 2018). The exercises were performed in supine position with a raised upper part of the body, in sitting or in standing position. Blood pressure, heart and breathing rate, saturation and electrocardiography were monitored. The terminating criteria for performing the exercises were in accordance the evidence-based 
recommendations for effective and safe diagnostic assessment and intervention strategies for the KT treatment of patients in intensive care units (Sommers, et al., 2015).

Exercise protocol for home-based exercise program Home-based KT was performed twice daily, every day, for 3 months. All the patients were given a device for inspiratory training and a booklet with instructions for exercising at home with a description of all therapeutic exercises and how to increase gradually the walking distance, stairs climbing, daily activities, etc. The patients were instructed how to measure their heart rate during the exercises at home. The indications for discontinuing the exercising are arrhythmia, tachycardia, precordial pain or chest discomfort, a change in the breathing pattern with inclusion of additional respiratory muscles, severe fatigue or dyspnea, muscle weakness, patient's desire to discontinue.

\section{Statistical analysis}

All statistical analyses were performed using SPSS for Windows, Version 19.0. The level of significance was set at $p$ less than 0.05 . All data subsets were assessed for the presence of a normal distribution (Shapiro-Wilk test $\mathrm{p}>0.05$ ) and for homogeneity with Levene's test for equality of variance before using parametric analysis. Differences between data in Six minutes walking test, Borg Scale and FTSTS were assessed by repeated measures analysis of variance with post hoc multiple comparisons by t-test with Bonferroni corrections.

\section{RESULTS}

The patients were observed over the study period of 3 months (Table 2). We compared the effect of KT in patients after TAVI on breathlessness, and functional strength of the lower and upper limbs in 4 time points.

Table 2. Changes in physical activity and dyspnea

(mean value and standard deviation) in the patients over the study period

\begin{tabular}{|c|c|c|c|c|}
\hline \multirow{2}{*}{ TEST } & \multicolumn{4}{|c|}{ mean values \pm SD } \\
\hline & before TAVI & on hospital discharge & $1^{\text {st }}$ month & $3^{\mathrm{d}}$ month \\
\hline Borg scale (points) & $6,6 \pm 2,6$ & $4,2 \pm 2,4$ & $2,8 \pm 1,6$ & $1,9 \pm 1,4$ \\
\hline $\mathrm{p}$-value & \multicolumn{4}{|l|}{$0,001^{\text {a }}$} \\
\hline FTSTS (sec) & $21,9 \pm 0,8$ & $20,8 \pm 0,2$ & $19,0 \pm 0,2$ & $17,1 \pm 0,2$ \\
\hline $\mathrm{p}$-value & \multicolumn{4}{|l|}{$0,009^{\text {a }}$} \\
\hline
\end{tabular}

TAVI-transcatheter aortic valve implantation; FTSTS-5 times sit-to-stand; ${ }^{a}-R M$ ANOVA; SD-standard deviation

The patients had decreased the levels of dyspnoea with 4,7 points in the mean values after 3 months of KT. Their score in mean values decreased from 6,6 points (very strong dyspnea) to 1,9 points (weak dyspnea). Improvement in the functional lower limbs muscle strength was observed because the time for performing the FTSTS test decreased with $4,8 \mathrm{sec}$ on the $3^{\text {rd }}$ month compared to the condition before TAVI in mean values. Both men and women improved their left and right hand grip strength in mean values. (Figure 1,2).

\section{Dynamometry of the women}

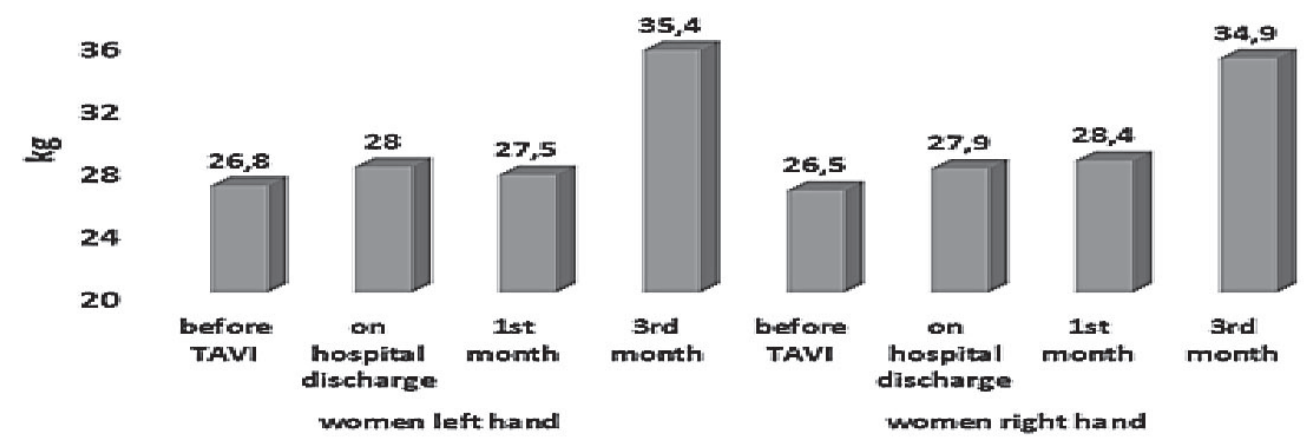

Figure. 1 Changes in the strength of the upper limbs in the mean values of the women 


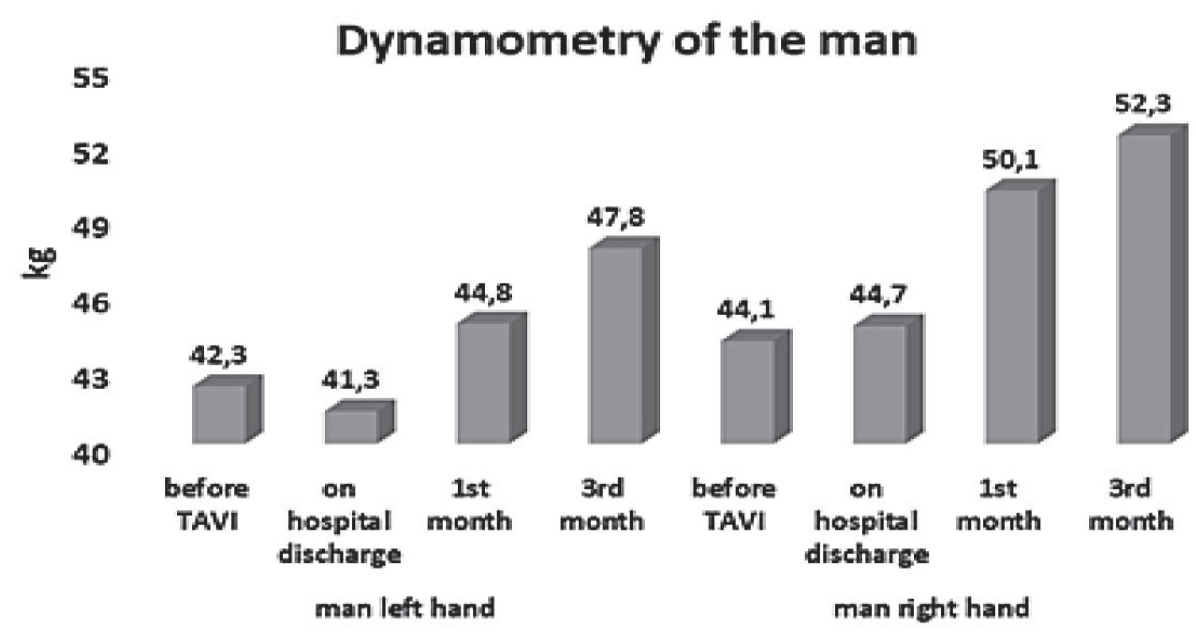

Figure 2. Changes in the strength of the upper limbs in the mean values of the men

The mean difference of the grip strength for the non-dominant left hand in women before TAVI and 1 month after was $0,7 \mathrm{~kg}$ and increased to nearly $8 \mathrm{~kg}$ on the $3 \mathrm{rd}$ month. The mean difference for the dominant right hand before TAVI and 1 month after was $1,9 \mathrm{~kg}$ and increased to $8,4 \mathrm{~kg}$ on the $3 \mathrm{rd}$ month. The mean difference of the grip strength for the non-dominant left hand in men before TAVI and 1 month after was $2,5 \mathrm{~kg}$ and increased to 5,5 $\mathrm{kg}$ on the $3 \mathrm{rd}$ month. The mean difference of the hand grip strength for the dominant hand before TAVI and 1 month after was $6 \mathrm{~kg}$ and increased to $8,2 \mathrm{~kg}$ on the $3 \mathrm{rd}$ month.

\section{DISCUSSION}

The role of the KT as a part of the clinical management of patients after TAVI is to maintain independency in daily life activities and participation in socio-cultural life (Russo, 2014).

The hand grip strength is a simple measure for screening and predicting important treatment outcomes such as length of in-hospital stay, complications after surgery, survival for elderly patients (Bohannon, 2008). Dominant hand grip strength is a marker that reflects frailty associated with incremental risks for late mortality. The prognostic value and optimal threshold of grip strength are not fully validated in elderly patients who undergo TAVI (Kagase, et al., 2018). There are different scales for average values depending on gender, age, dominant hand or body mass index. According to the hand grip strength average tables the mean value for men between 70-99 years old is $21,3-35,1 \mathrm{~kg}$ and for women between 70-99 years old is 14,7-24,5 $\mathrm{kg}$ (https://www.prohealthcareproducts.com/blog/ hand-grip-strength-norms/). In another study the grip strength was assessed as the average of 3 trials of maximal isometric grip measured in kilograms using a Jamar dynamometer (Sammons Preston, Chicago, IL). Grip strength was considered weak if it was $<17,3 \mathrm{~kg}$ for women with BMI between 23,126 and $<30 \mathrm{~kg}$ for men with BMI between 24,1-28 (Fried, et al., 2001). A study conducted in Sweden reported the mean average values for healthy men 75-79 years of age for the dominant hand to be 36,8 $\mathrm{kg}$ and for the non-dominant hand to be $36,6 \mathrm{~kg}$. The average mean values for healthy women 75-79 years of age are $25,0 \mathrm{~kg}$ for the dominant hand and $23,7 \mathrm{~kg}$ for the non-dominant hand (Werle, et al., 2009). The grip strength is considered to be a reflection of overall muscle mass and fitness in adults, and it is an indicator of muscle strength in aging people. Many authors recommend the importance of grip strength assessment before and after TAVI (Kagase, et al., 2018; Shimura, et al., 2017). The results of the current study demonstrated that peak grip strength was a useful marker for predicting all-cause mortality following TAVI, particularly the non-cardiac related deaths (Shimura, et al., 2017). In a recent large study with 281 men and 646 women after TAVI the grip strength was measured with a conventional dynamometer before TAVI, with the patient in a standing position with an arm extended straight down to the side. The mean peak grip strength was $17,9 \pm 6,9 \mathrm{~kg}$ in the overall cohort, $24,5 \pm 7,0 \mathrm{~kg}$ in men, and $15,0 \pm 4.3 \mathrm{~kg}$ in women (Kagase, et al., 2018). The grip strength of our patients is above the average values for gender, age, dominant and non-dominant hand and body mass 
index.

The FTSTS test is a simple measure to assess the lower limb muscle strength and the risk of falls. Average values are 11,4 $\mathrm{sec}$ (60-69 years), 12,6 sec (7079 years) and $14,8 \mathrm{sec}$ (80-89 years) (Bohannon, 2006). Patients with results over $15 \mathrm{sec}$ are considered to have an increased risk of falling. A change of $2.3 \mathrm{sec}$ is significant (Bohannon, 2006). Our patients' results decreased significantly from $21,9 \pm 0,8$ sec to $17,1 \pm 0.2 \mathrm{sec}$ in mean values but the risk of falling still remained.

\section{CONCLUSION}

The applied three-month KT program benefits all participating patients' upper and lower limbs muscle strength compared with their baseline results and decreased dyspnea.

\section{REFERENCES}

Bohannon, R. (2006). Reference values for the five-repetition SIT-TO-STAND TEST: A descriptive META-ANALYSIS of data from elders. Perceptual and Motor Skills, No.103; pp. 215-222

Bohannon, R. (2008). Hand-Grip Dynamometry Predicts Future Outcomes in Aging Adults. Journal of Geriatric Physical Therapy, Vol 31 No.1, pp. 3-10

Borg, G. (1982). Psychophysical bases of perceived exertion. Med Sci Sports Exerc., No.14, pp. 377-381.

Fauchere, I., Weber, D., Maier, W. et al. (2014). Rehabilitation after TAVI compared to surgical aortic valve replacement. International Journal of Cardiology, Vol 173 No.3, pp.564-566

Fried, L., Tangen, C., Walston, J., et al. (2001). Frailty in older adults: evidence for a phenotype. $J$ Gerontol A Biol Sci Med Sci., No.56, pp.146-156.

Grigorova-Petrova, K. (2018). Vliyanie na inspiratornata trenirovka vurrkhu podvizhnostta na diafragmata pri patsienti sŭs sledinsultni sŭstoyaniya. Sport i nauka, No.6, pp. 95-103
Kagase, A., Yamamoto, M., Shimura, T., (2018). Sex-Specific Grip Strength After Transcatheter Aortic Valve Replacement in Elderly Patients. JACC: Cardiovascular interventions, Vol. 11, NO. 1, pp. 100-103

Ribeiro, G., Melo, R., Deresz, L. et al. (2017). Cardiac rehabilitation programme after transcatheter aortic valve implantation versus surgical aortic valve replacement: Systematic review and meta-analysis. European Journal of Preventive Cardiology, 0(00) 1-10 DOI: $10.1177 / 2047487316686442$

Ross, R., Murthy, J., Wollak, I., Jackson, A. (2010). The six minute walk test accurately estimates mean peak oxygen uptake. BMC Pulm Med., No.10, pp. 31-40.

Russo, N., Compostella, L., Tarantini, G., et al. (2014). Cardiac rehabilitation after transcatheter versus surgical prosthetic valve implantation for aortic stenosis in the elderly. European Journal of Preventive Cardiology, Vol 21 No.11, pp.1324-1331

Scottish Intercollegiate Guidelines Network (SIGN). Cardiac rehabilitation. Edinburgh: SIGN; 2016. (SIGN publication no. 150). [July 2017]. http://www.sign.ac.uk Shimura, T., Yamamoto, M., Kano, S., et al., (2017). Impact of the Clinical Frailty Scale on Outcomes After Transcatheter Aortic Valve Replacement. Circulation, Vol 135, pp. 2013-2024

Shimura, T., Yamamoto, M., Kano, S., et al. (2017). Impact of frailty markers on outcomes after transcatheter aortic valve replacement: insights from a Japanese multicenter registry. Ann Cardiothorac Surg., Vol 6 No. 5, pp. 532-537.

Sommers, J., Engelbert, R., Dettling-Ihnenfeldt, D., Rik Gosselink, R., et al. (2015). Physiotherapy in the intensive care unit: an evidence-based, expert driven, practical statement and rehabilitation recommendations. Clin Rehabil., Vol 29 No.11, pp. 1051-1063.

Werle, S., Goldhahn, J., S Drerup, S., et al. (2009). Ageand Gender-Specific Normative Data of Grip and Pinch Strength in a Healthy Adult Swiss Population. Journal of Hand Surgery, Vol. 34E No. 1, pp. 76-84

https://www.prohealthcareproducts.com/blog/handgrip-strength-norms/ 